\title{
LOCAL ELASTIC STRESS APPROACH FOR FATIGUE LIFE CALCULATION BY THE FINITE ELEMENT METHOD (FEM)
}

The paper discusses possibilities of a description of material fatigue and prediction of service life of machine parts on the basis of stress state calculations using the finite element method (FEM). It focuses on the local approach to the assessment of service life that is based on the determination of elastic stress peaks in stress raisers (notches) and on the derivation of the fatigue stress curves for assessed structural points. The method of taking into account the stress concentration and stress gradient during the determination of synthetic fatigue curves is described. Furthermore, methods of processing the loading history and correcting mean stress values of loading cycles are described.

\section{Introduction}

Material fatigue is one of the important limiting states of structures, during which a fatigue crack can be initiated in a local material volume as a consequence of repeated cyclic loading leading to an undesirable fracture of the entire load bearing cross section of a machine part. At present, the finite element method (FEM) is a standard method for assessing states of structural deformation and stress. There are several commercially available FEM software packages and modules for the subsequent assessment of the fatigue damage can also be purchased. The goal of this paper is to demonstrate certain relations between individual approaches to the assessment of the fatigue service life with the help of the FEM modules and to describe in detail an approach based on local values of elastic stress in notches.

\section{Various approaches to the assessment of material fatigue and service life of machine parts}

Today, the method based on the elasticity theory and analytical calculations of the stress of mechanical parts and structures can be denoted as a classical approach. This nominal stress approach (NSA) is based on the classification of stress states (tensile stress, bending stress, local stress, etc.) and on the calculation of the stress amplitudes and mean stress values in the assessed critical cross section of a machine part. It assumes the knowledge of the fatigue stress curve ( $S-N$ curves) at this point or at least its qualified estimate. If the values of the stress amplitude exceed the fatigue limit, $\sigma_{C}$, determined for this curve, a limited service life of the machine part can be expected. As operational conditions are frequently characterized by a stochastic time course, it is first necessary to obtain single-parametric or multiple-parametric histograms of the stress incidence frequency. At present, the rain flow method and its two-parametric output of the frequency of the incidence of cycles, $n_{i}$, into a rain flow matrix, is the method used exclusively in practice for the analysis of loading history. The magnitude of fatigue damage, $D$, for the loading spectrum processed by this method, is evaluated in most cases using the Palmgren and Miner rule and its modification. It holds that $D=\sum_{i} \frac{n_{i}}{N_{i}}$ for the basic form of this rule, where $N_{i}$ is the limit number of cycles determined from the $S-N$ curve. In order to improve the accuracy of the calculations for a new generation of products, the results of the experimental tests of the service life of the preceding generation can be used and the rule can be corrected using the ratio of the actual and calculated fatigue damage (the so-called relative Palmgren and Miner hypothesis).

The main disadvantage of NSA is its complicated application in the FEM calculations, because it requires assessment of individual categories of nominal stress values in the assessed cross sections. For this reason, the so-called local approaches are applied. These approaches are based on a thesis that the time until the initiation of a fatigue crack at a critical local point (usually at the root of sharp geometrical notches) is the same as the fatigue life of a sample without notches that is loaded by stress parameters and deformations at a critical local area. These parameters can be determined by the FEM calculations. In the region of a low-cycle fatigue, i.e. approximately up to a service life of $N=10^{4}$ up to $10^{5}$ cycles, where the plastic component of the relative deformation should be taken into account, the local plastic stress and strain approaches (LPSA) are used. These methods are based on the use of a strain life curve and damage parameter, $P$, that is determined according to the local quantities in the notch. The $P_{S W T}$ damage parameter according to Smith, Watson and Topper is used in most cases. These approaches were compared, for example, in Ref. [1]. The disadvantage of these methods is that it is necessary to solve the elastic and plastic problem using FEM or to apply the analytical formulae for the adaptation of the elastic local parameters to the elastic and plastic solutions (the Neuber hyperbola method, the method of equivalent stress energies, etc.).

\footnotetext{
* Milan Růžička

Czech Technical University in Prague, Faculty of Mechanical Engineering, Technická 4, 16607 Prague 6, Czech Republic.

Telephone: +420 224352 512, Fax: +420 233322 482, E-mail: milan.ruzicka@fs.cvut.cz
} 
In the region of a high-cycle fatigue, it is sufficient to use the local elastic stress approach (LESA). The advantage of this approach is that the direct FEM calculations can be used in the elastic region of deformations, which makes it possible to use the superposition of stress values during various loading states of the structure. Some of the most important aspects of this method will be discussed in the following paragraphs, where the method will be compared with the nominal approach.

\section{Stress concentration and the stress gradient effect in the notches}

It is known that the concentration of nominal, $\sigma_{n}$, stress to the local elastic (virtual), $\sigma_{f i c}$, stress intensities which are at the root of geometrical notches can be described using a shape factor defined as $K_{t}=\frac{\sigma_{f i c}}{\sigma_{n}}$. The magnitude of the so-called exposed material volume in which a significant part of the local damage occurs, can be described using the relative stress gradient at the notch root, $G$, where

$$
G=\left[\frac{\Delta \sigma_{y}}{\Delta x}\right]_{x \rightarrow 0} \cdot \frac{1}{\sigma_{f i c}}\left[\mathrm{~mm}^{-1}\right] .
$$

If the stress peaks occur along the entire $L$ region (as, for example, in Fig. 1, where $L$ is the sample thickness), the $L / G$ ratio represents the exposed region. For the etalon sample, the ratio of these quantities defines the similarity factor, $\theta=\frac{L / G}{L_{0} / G_{0}}$, of the exposed volume.

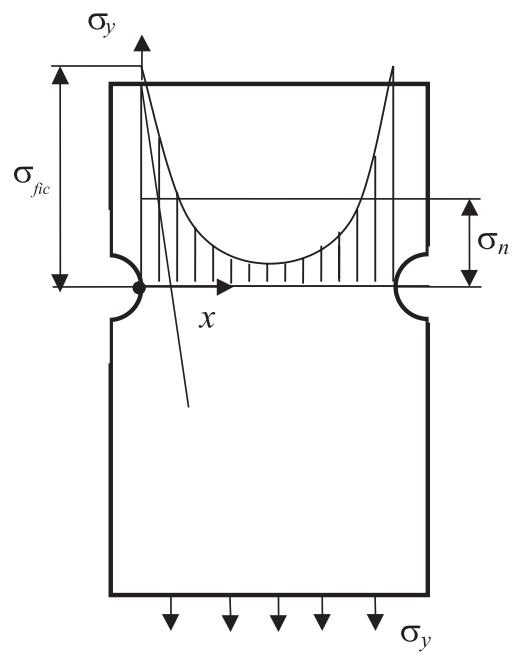

Fig. 1

All these quantities can be determined in the notch locality from the results of the FEM calculations, also during complex loading conditions, when the condition of the multicomponent strain is usually assessed using equivalent stress values according to strength hypotheses, for example, $\sigma_{H M H}$ according to Huber, Mises and Hencky.

\section{The effective notch effect on the fatigue strength}

It is also known that the effect of the stress peaks on the notch fatigue strength is not as significant as it would correspond to the theoretical stress concentration. Experiments define the effective notch effect on the fatigue limit by a notch factor, $K_{f}=\frac{\sigma_{C}}{\sigma_{C}^{*}}$. In the past, several relations were proposed for the computational estimation of the notch factor, which are reviewed, for example, in Ref. [2]. Let us define the ratio of the shape and notch factor by the fatigue ratio, $n=\frac{K_{t}}{K_{f}}$. The methods for expressing the $n$ quantity can be split into two major groups. The first group is formed by relations that are determined in dependence on the notch root radius, $\rho$. The second group involves expressions depending on the magnitude of the relative stress gradient, $G$. In Table 1 , the values of the $n_{\rho}$ and $n_{G}$ ratio are indicated for some most frequently used relations.

For the FEM applications, the expression by means of the stress gradient, $G$, turns out to be more convenient. For the determination of the local fatigue limit in the notch root, $\sigma_{C, F E M}$, (i.e., of the limit values of the elastic stress peaks in the FEM calculations), the following relation can be used:

$$
n=\frac{K_{t}}{K_{f}}=\frac{K_{t} \cdot \sigma_{C}^{*}}{\sigma_{C}}=\frac{\sigma_{C, F E M}}{\sigma_{C}} \text {, so that } \sigma_{C, F E M}=n_{G} \cdot \sigma_{C},
$$

where $\sigma_{C}$ is the material fatigue limit during a homogeneous uniaxial tensile stress.

Analogously to the $K_{f}$ factor introduced in the region of the permanent fatigue strength, it is possible to define the notch factor, $K_{f, N}=\frac{\sigma_{A}}{\sigma_{A}^{*}}$, in the region of the limited life from the fatigue test results or, as the case may be, the fatigue ratio by the relation $n_{G, N}=\frac{K_{t}}{K_{f, N}}$. It is then possible to obtain the fatigue curve of virtual stress values at the notch root, $\sigma_{F E M}=\frac{K_{t}}{K_{f, N}} \cdot \sigma_{C}=n_{G, N} \cdot \sigma_{C}$, which must lie above the smooth sample curve (see Fig. 2). In practical calculations, we can also use an opposite procedure. During the damage calculation, we use the fatigue curve of a smooth sample and correct the local stress amplitudes by calculating the quantity $\sigma_{c o r}=\sigma_{F E M} \cdot \frac{K_{f, N}}{K_{t}}$.

This procedure is also illustrated in Fig. 2. 
Table 1

\begin{tabular}{|l|l|l|l|}
\hline \multicolumn{1}{|c|}{ Author } & Fatigue ratio $\mathrm{n}$ & Note & Equation \\
\hline Neuber & $n_{\rho}=1+\frac{A}{\rho} \cdot\left(1-\frac{1}{K_{f}}\right)$ & $\begin{array}{l}\text { where } A=f\left(R_{m}\right) \text { is the } \\
\text { Neuber factor, see, e.g., [2] }\end{array}$ & (2) \\
\hline Peterson & $n_{\rho}=1+\sqrt{\frac{a}{\rho}} \cdot\left(1-\frac{1}{K_{f}}\right)$ & where $a$ is the critical surface layer depth, see, e.g., [2] & empirical factor $a^{\prime}$ see, e.g... [2] \\
\hline Heywood & $n_{\rho}=1+2 \sqrt{\frac{a^{\prime}}{\rho}} \cdot\left(1-\frac{1}{K_{f}}\right)$ & parameter $c$ see, e.g., [2] & (3) \\
\hline Siebel, Stiller & $n_{G}=1+\sqrt{c \cdot G}$ & where $R_{e}$ is the yield strength \\
\hline Bäumel Seeger [3] & $n_{G}=1+\sqrt{G} \cdot 10-\left(\frac{R_{e}}{810}+0.35\right)$ & $\begin{array}{l}\text { where } \sigma_{C, b} \text { and } \sigma_{C} \text { are the bending and tensile fatigue limits, } \\
d \text { is the diameter of the bending sample }\end{array}$ & (6) \\
\hline Eichelseder [4] & $n_{G}=1+\left(\frac{\sigma_{C, b}}{\sigma_{C}}-1\right) \cdot\left(\frac{G}{2 / d}\right)^{k}$ & $\begin{array}{l}\text { here } v_{\infty} \text { is the magnitude factor for the homogeneous strength, } \\
\mu \text { is an exponent }\end{array}$ & (7) \\
\hline Volejnik, Kogaev [5] & $n_{G}=1+\left(\frac{1}{v_{\infty}}-1\right) \cdot\left(\frac{L / G}{L_{0} / G_{0}}\right)^{\mu}$ & \\
\hline
\end{tabular}

\section{Synthetic S-N curves}

Fig. 2 indicates the analogy between the LESA approach and the NSA approach. While the fatigue curve is corrected in the downward direction in the nominal approach with respect to the notches, and the fatigue damage

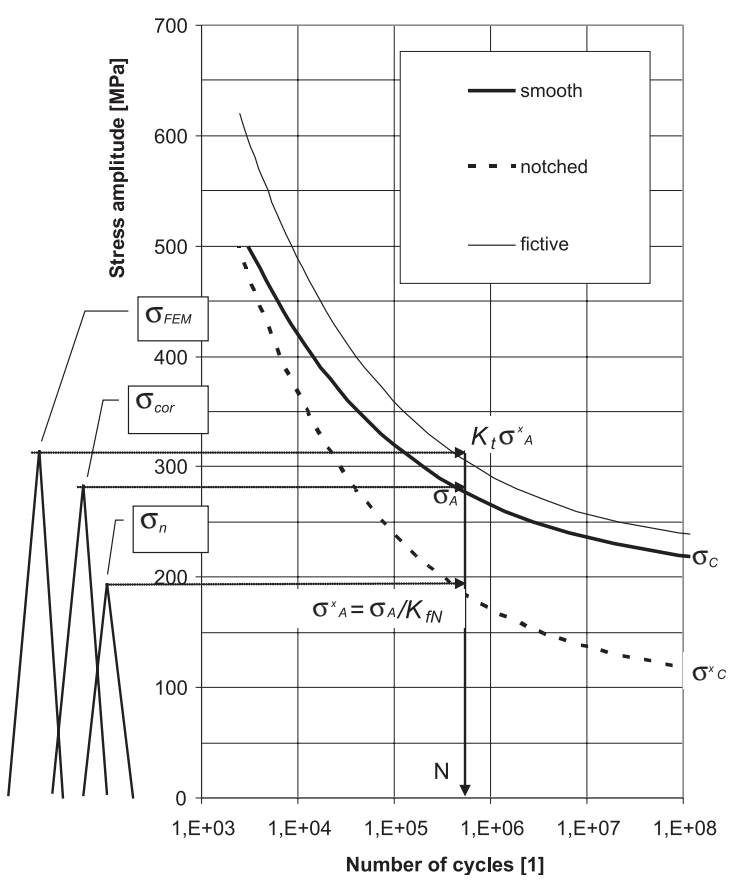

Fig. 2 is determined from the nominal stress amplitude, the local approach uses the corrected stress peak at the notches and the

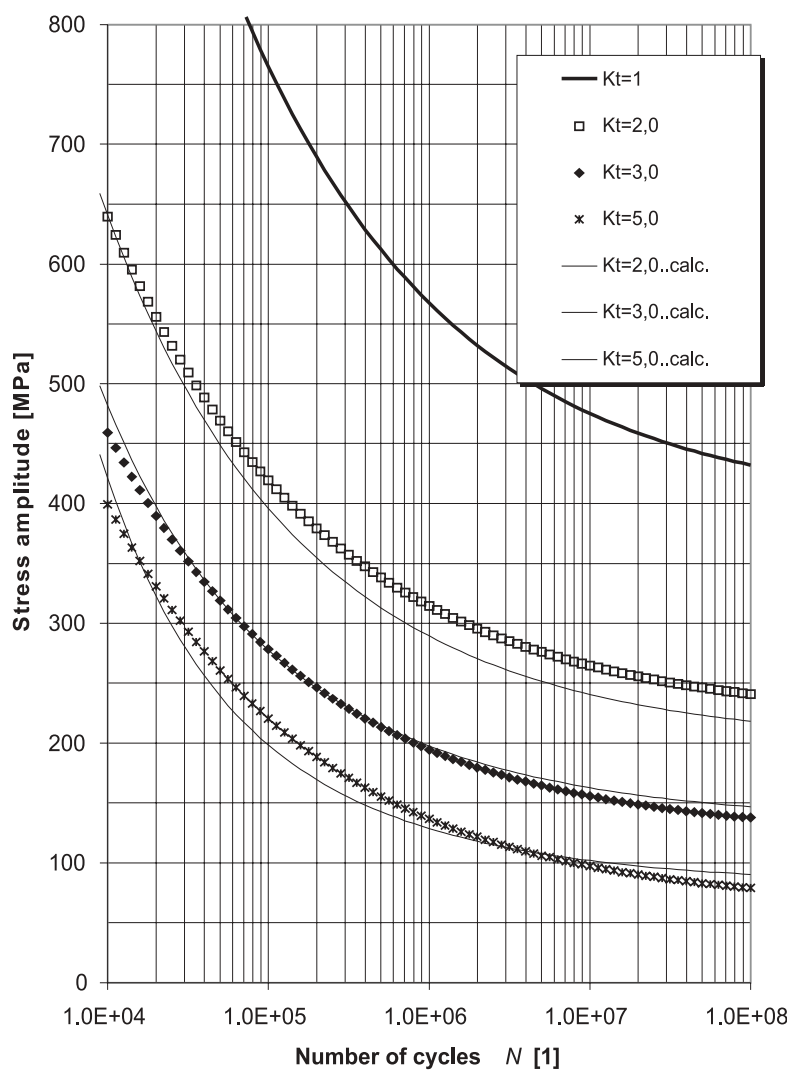

Fig. 3 
initial fatigue curve of the sample without notches. Let us mention, however, that all the effects of the surface quality of the actual machine part should be projected into the curve and, as the case may be, its further technological modifications. The magnitude factor is taken into account in the above-indicated similarity criterion of the stress gradient effect and exposed volume.

The author of this paper proposed a modification of the Heywood expression (see [6]) for the description of the notch effect in the region of the limited life, i.e., for the expression of the $K_{f, N}$ coefficient, $K_{f, N}=1+\left(1-K_{t}\right) \cdot \mu(N)$,

in which the dependence on the relative stress gradient, $\mathrm{G}$, was expressed in the following form: $n_{G}=\frac{K_{t}}{K_{f}}=1+\sqrt{G} \cdot 10^{-(K 1)}$ and the time functions as $\mu(N)=\frac{(\log N)^{E}}{B+(\log N)^{E}}$ with the parameters $E=4 \cdot G^{K 2}$ and $B=\left[\frac{1}{(1+G)^{K 3}} \cdot \frac{K 4}{R_{m}}\right]^{2}$.

The curves of ASTM 300M steel according to MIL-HDBK catalogue [7] are taken for an example here. The mathematical description of fatigue curves and of their parameters for smooth and specific notched specimens with a variable shape factor $K_{t}$ are presented in Tab. 2.

Fig. 3 depicts the comparison of commented catalogue curves and the derived ones. The goal is to obtain the smallest possible difference in the area of middle and higher notch factor and in the range of cycles between $10^{4}$ and $10^{7}$ cycles. It can be clearly seen that the coincidence is satisfactory - especially towards the original sources, where the significant scatter of experimental points is clear, and to the fact, that the punctuality of result approximation by a mean curve is affected with certain error. The parameters of a studied approximation are given in the last column of Tab. 2. To describe the effect of number of cycles (i.e. the $\mu(N)$ factor) on the size of different notch coefficient $K_{f}$, the $K_{f, N}$ dependencies were depicted (Fig. 4). It can be traced that the notch factor pro- gressively decreases with higher concentrations on the specimen for lower numbers of cycles. A bunch of the so-called synthetic fatigue curves can be generated for any general stress gradient and shape factor; these curves can represent the required areas of the structure in the NSA approach. The example of these curves is indicated in Fig. 5.

The set of these equations can also be used for correcting the elastic stress peaks according to relation (8) in the LESA approach.

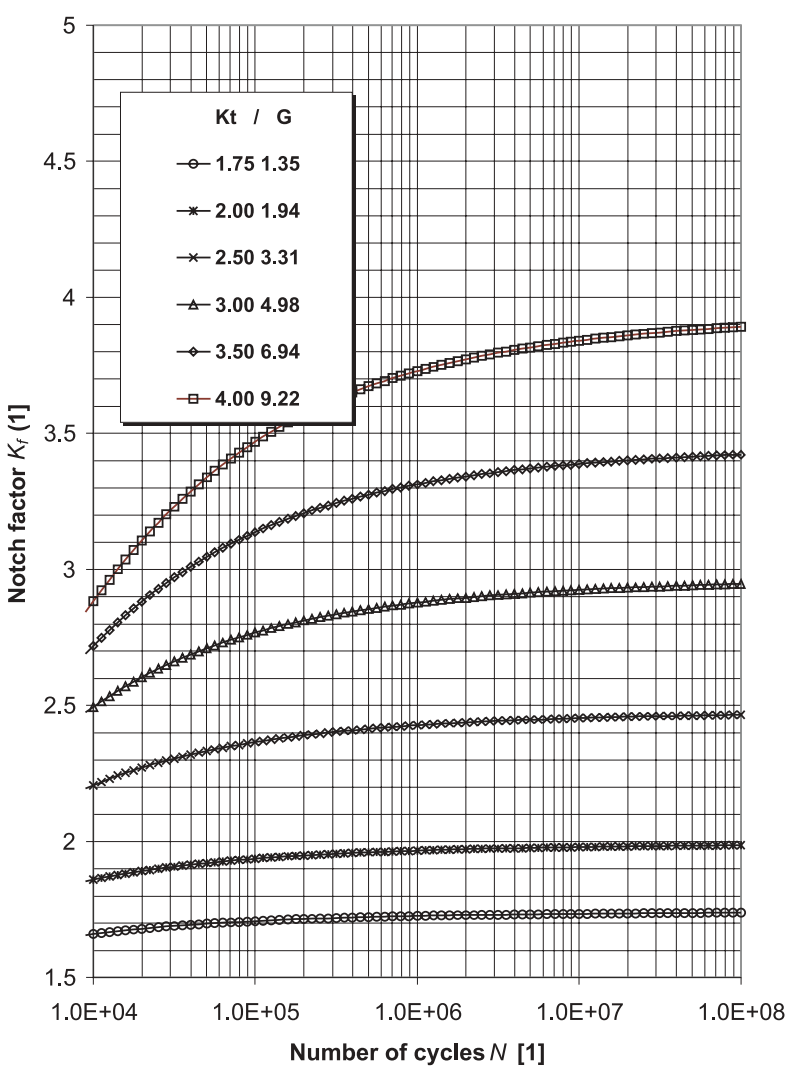

Fig. 4

Table 2

\begin{tabular}{|c|c|c|c|c|}
\hline \multirow{6}{*}{$\begin{array}{c}300 \mathrm{M} \\
\text { steel }\end{array}$} & $\begin{array}{c}\text { Shape } \\
\text { factor } K_{t}\end{array}$ & $\begin{array}{l}\text { Fatigue curve description } \\
N \cdot\left(\sigma_{\text {max }, e g}-\sigma_{C}\right)^{w}=C\end{array}$ & $\begin{array}{c}\text { Exponent } \mathrm{p} \\
\sigma_{\text {max }, e g}=\sigma_{\max }(1-R)^{p}\end{array}$ & $\begin{array}{r}\text { Synthetic curve } \\
\text { parameters } K_{i}\end{array}$ \\
\hline & 1.0 & $\log N=10,58-3,02 \cdot \log \left(\sigma_{e q}-75,0\right)$ & 0,39 & $K 1=2.409$ \\
\hline & 2.0 & $\log N=12,87-5,08 \cdot \log \left(\sigma_{e q}-55,0\right)$ & 0,36 & $K 2=0.1$ \\
\hline & 3.0 & $\log N=9,52-3,00 \cdot \log \left(\sigma_{e q}-25,0\right)$ & 0,50 & $K 3=-0.989$ \\
\hline & 5.0 & $\log N=9,61-3,04 \cdot \log \left(\sigma_{e q}-15,0\right)$ & 0,52 & $K 4=6100$ \\
\hline & \multicolumn{2}{|c|}{$\begin{array}{l}N . . . \text { lifetime in number of cycles } \\
\sigma_{\text {max }, \text { eq }} \text { upper stress of equivalent cycle [ksi] } \\
\sigma_{\max } \ldots \text { upper stress of loading cycle [ksi] } \\
\sigma_{c} \ldots \text { fatigue limit [ksi] }\end{array}$} & \multicolumn{2}{|c|}{$\begin{array}{l}p \ldots \text {.. exponent for equivalent stress } \\
C, w \ldots \text { fatigue curve parameters } \\
R \ldots \text { coefficient of cycle asymmetry } \sigma_{\min } / \sigma_{\max } \\
\text { Remark: } 1 \mathrm{ksi}=6.894 \mathrm{MPa}\end{array}$} \\
\hline
\end{tabular}




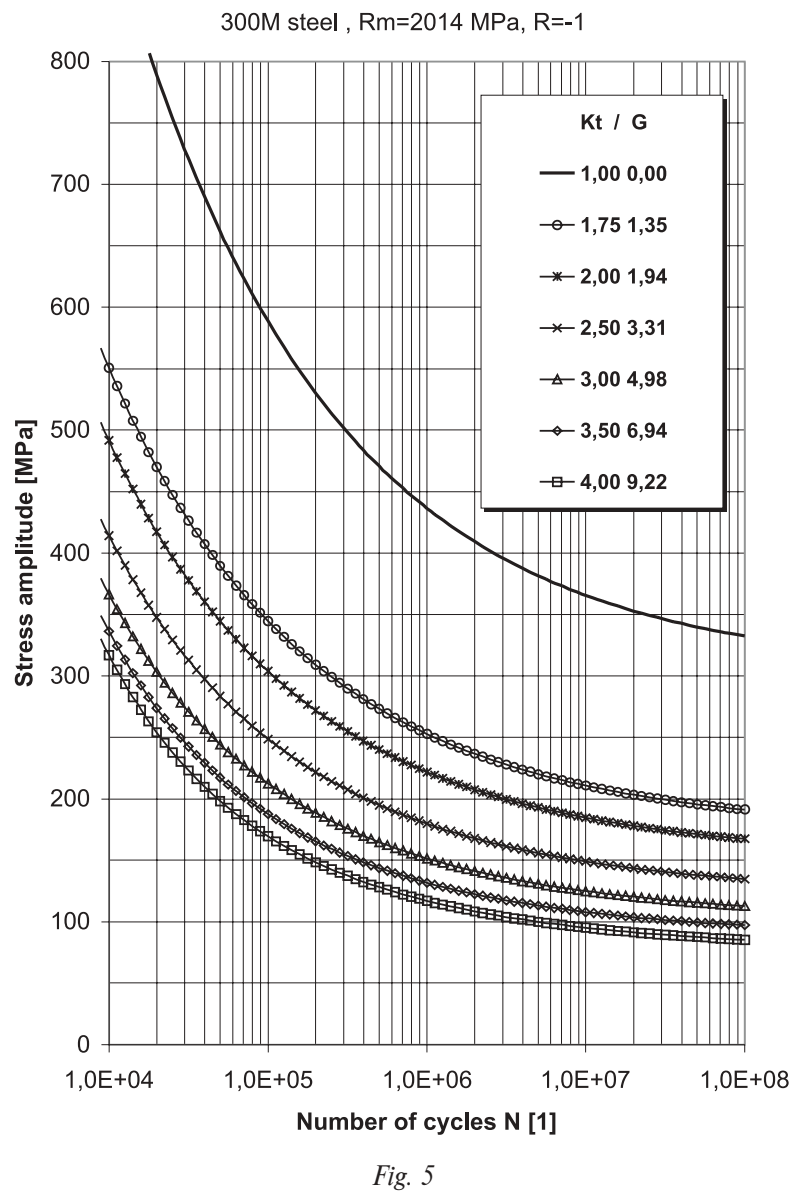

\section{Correction for the effect of the mean stress}

For the purposes of the calculation of the fatigue damage, the biparametric description of the loading history stored in the rain flow matrix should be transformed to the equivalent stress cycle. This means that for a general stress cycle of amplitude $\sigma_{a}$ and mean value $\sigma_{m}$, described also by the asymmetry factor $R=\frac{\sigma_{\min }}{\sigma_{\max }}$,

we assign a cycle with the same damaging effect. Let us mention that the correction is carried out only for the region of tensile mean stress values. Usually, the equivalent symmetrically alternating cycle is assigned (i.e., for $\sigma_{m}=0$ or $R=-1$ ) and denoted as $\sigma_{a, e q}$, or the repeated cyclic load (i.e., for $\sigma_{m}=\sigma_{a}$ or $R=0$ ) is assigned and denoted as $\sigma_{\text {max }, e q}$. For the determination of the equivalent cycle, it is possible to use for example the relations for the approximation of the Haigh diagram. We obtain $\sigma_{a, e q}=$ $=\sigma_{a} \frac{1}{1-\left(\frac{\sigma_{m}}{\sigma_{f}}\right)^{s}}$, where $\sigma_{j}$ represents the static fracture strength and the $s$ exponent describes both the linear form of the limit curve (for $s=1$ ), and for example the parabolic form (for $s=2$ ). The behaviour of the actual machine parts with stress raisers of the geometrical and technological type (for example, parts with welds) revealed that the initial significant reduction of the fatigue ultimate strength decreases with increasing prestressing and that the limit curve has a nonlinear form. In Ref. [7], the limit curve of the Haigh diagram is indicated in the form of $\sigma_{a}^{p} \cdot\left(\sigma_{a}+\sigma_{m}\right)^{1-p}$, from which the equivalent amplitude can be expressed as

$$
\sigma_{a, e q}=\sigma_{a}^{p} \cdot\left(\sigma_{a}+\sigma_{m}\right)^{1-p}=\sigma_{a}\left(\frac{2}{1-R}\right)^{1-p}
$$

For metallic materials (carbon steels, aluminium alloys), the value of the $p$ exponent is close to $p=0.5$, so that relation (10) becomes

$$
\sigma_{a, e q}=\sqrt{\sigma_{a} \cdot\left(\sigma_{a}+\sigma_{m}\right)}=\sqrt{2} \cdot \sigma_{a}-\frac{1}{\sqrt{1-R}}
$$

The value of the repeated cyclic load can be expressed from Eq.(10) analogically as

$$
\sigma_{\max , e q}=\sigma_{\max }(1-R)^{p},
$$

which for $p=0.5$ takes the form

$$
\sigma_{\max , e q}=\sigma_{\max } \sqrt{(1-R)}=\sqrt{2 \sigma_{a} \cdot\left(\sigma_{a}+\sigma_{m}\right)} .
$$

Relation (13) is known for example in the aerospace engineering as the Oding correction. The correction according to Eq.(11) can also be considered as a certain analogy; this correction forms the basis for the calculation of the damage parameter, $P_{S W T}$, according to Smith, Watson and Topper, as already mentioned in the introductory part. It holds for this parameter that $P_{S W T}=\sqrt{\sigma_{a} \cdot\left(\sigma_{a}+\sigma_{m}\right)}=\sqrt{E \cdot \varepsilon \cdot\left(\sigma_{a}+\sigma_{m}\right)} ;$ in the low-cycle fatigue region, the local elastic and plastic stress and the deformation amplitudes are considered in this expression.

\section{Scheme of the LESA application and conclusion}

The above-discussed aspects of the fatigue damage can be summarized in the following proposal of the procedures for the prediction of the fatigue life of actual machine parts of structures subject to FEM calculations in the elastic stress region. The input quantities of the calculation are:

- The results of the stress state determined using FEM for the individual states of the effect of operational forces (usually for a "unit" load).

- The loading history corresponding to a given unit of the operational time obtained by a linear combination of individual loading states and processed by the rain flow method.

- The fatigue curve of the material used that is modified with respect to the surface layer quality.

According to the scheme indicated in Fig. 6, a relation can be shown to exist between the following steps of a further procedure:

- Correction of the elastic stress peaks according to Eq. (8)

- Correction for the mean stress of the loading cycle.

- Application of the rules for damage accumulation, including the correction of the results of the calculation on the basis of 
the carried out fatigue tests during the simulation of the operational loading spectra.

The result is interpreted as the fatigue life until the initiation of a fatigue crack at a local point of the structure. During the computer processing of each point of the surface of a machine part, a map of fatigue damage can be displayed. This makes it possible to find quickly the critical points visually and the lengths of the predicted fatigue life by their mutual comparison, analogously as during the assessment of the stress state during a static analysis by means of FEM. Some examples of applications were already published; see e.g. Refs. [8] and [9].

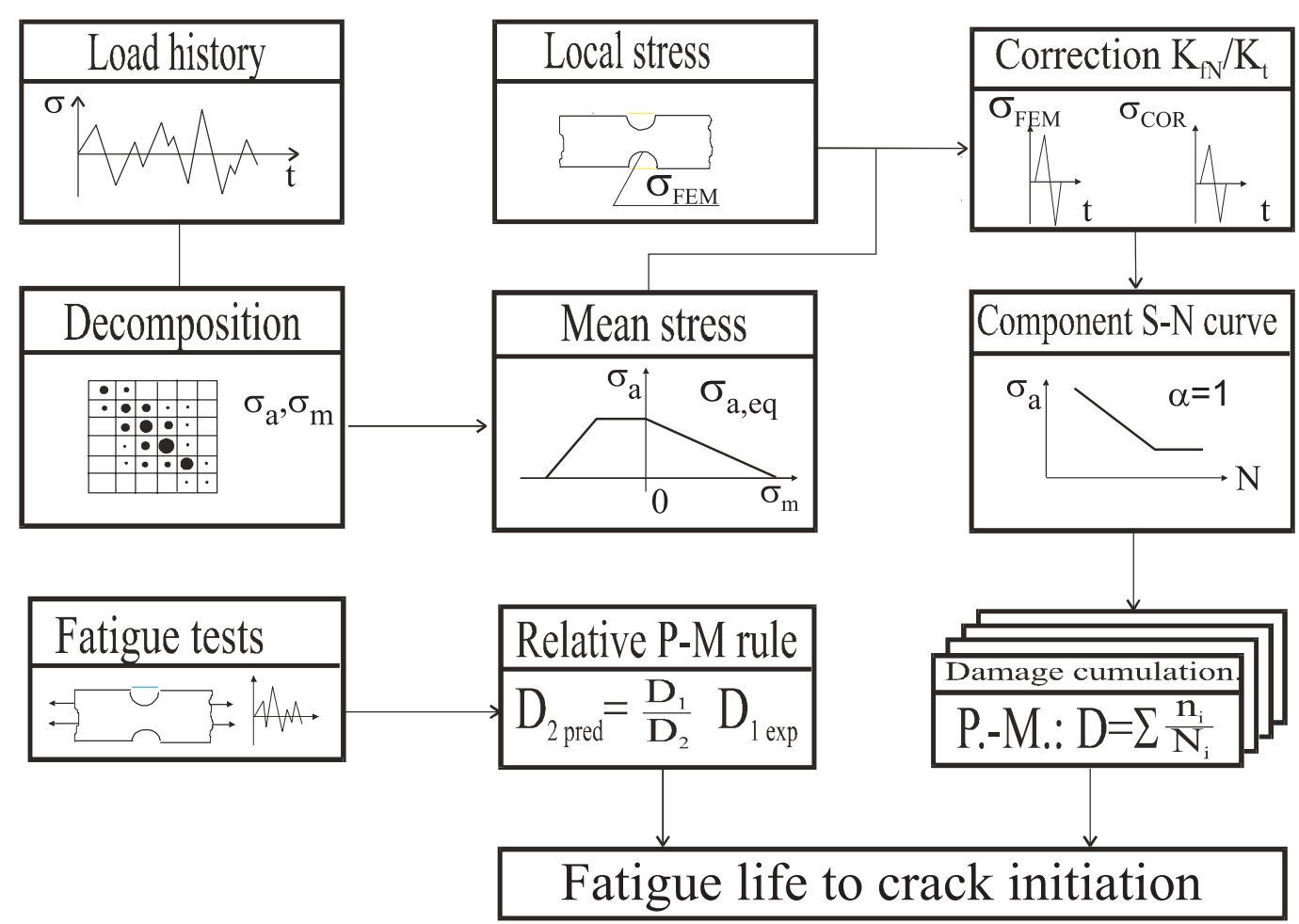

Fig. 6

\section{References}

[1] RŮŽIČKA, M.: Life Prediction of Machine Parts Using the Local Approach. (in Czech). In: Dynamic of Machines '94. Prague, Institute of Thermomechanics AS CR. 1994. pp. 77-80.

[2] RŮŽIČKA, M., HANKE, M., ROST, M.: Dynamic Strength and Service Life. (in Czech), lecture notes CTU Prague, 1992.

[3] BÄUMEL, A., SEEGER, T.: Material Data for Cyclic Loading - Suppl. 1. Materials Science Monographs 61, Elsevier Sc. Publisher, Amsterdam 1990.

[4] EICHELSEDER, W., LEITNER, H.: Influence of Stress Gradient on S/N-Curve. In: New trends in fatigue and fracture, Metz April, 2002.

[5] VOLEJNIK, N, V.: Carrying Capacity of Machine Parts. (in Russian). Scientific Idea Publ., Moscow 1985.

[6] HEYWOOD, R. B.: Design Against Fatigue, Pergamon Press, 1965.

[7] Military Standardization Handbook: Metallic materials and elements for aerospace vehicle structure. MIL-HDBK-5D, 1987.

[8] RÜŽIČKA, M.: Service Life Prediction of the Airplane Engine Bed. (in Czech). In: Dynamic of Machines 99, Institute of Thermomechanics CAS, Prague 1999, pp. 217-224. ISBN 80-85918-48-X.

[9] RŮŽIČKA, M., PAPUGA, J., KUBA, P.: Integration of Methods of the Experimental Stress Analysis in Design Mountainbike Components. In: 17th Danubia Adria Symphosium - Extended Summariers. Department of Mechanics, CTU Prague 2000, p. $293-296$. ISBN 80-01-02234-X. 